\title{
Sitzung vom 10. Mai 1915.
}

Vorsitzender: Hr. E. Beckmann, Vizepräsident.

Nachdem die Protokolle der Generalversammlung und der Gesellscbafts-Sitzung vom 26. April genehmigt sind, verliest der Schriftführer den weiter unten abgedruckten Auszug aus dem Protokoll der Vorstandssitzung vom gleichen Tage.

Als außerordentliche Mitglieder werden vorgeschlagen die HHrn.: Huss, Richard, Rappstr. 17, Tübingen (durch C. Bülow R. Weinland);

Tausz, Dr.-Ing., E., Pécs (Ungarn) (diurch C. Engler und H. Franzen);

Kautsky, H., Seesenerstr. 20, Berlin-Halensee (durch A. Rosenheim und R. J. Meyer);

Kobelt, Dr. pbil. V., Haberlandstr. 3, Berlin W. 30 (durcb E. Hoffmann und M. K. Holfmann).

Für die Bibliothek sind als Geschenke eingegangen:

$2167 \mathrm{c}$. Söderbaum, H. G., Jac. Berzelius Lettres. IV.: Correspondance entro Berzelius et P. L. Dulong (1819-1837). Upsala 1915.

2296. Binz, A., Technologische Vorträge. Berlin 1910/15.

In der Sitzung wurde folgender Yortrag gebalten:

I. Traube: Über Farbstolle. - Vorgetragen vom Verfasser.

Der Vorsitzende:

E. Beckmanu.
Der Schriftührer:

F. Mylius.

\section{Auszug aus dem \\ Protokoll der Vorstandssitzung vom 26. April 1915.}

Anwesend die HHrn. Vorstandsmitglieder: E. Beckmann, C. Duisberg, E. Fischer, S. Gabriel, K. A. Holmann, B. Lepsius, W. Marckwald, C. A. v. Martius, F. Mylius, F. Oppenheim, A. Rosenbeim, W. Traube, H. Wichelbaus, W. Will, ferner der Leiter der Abteilung für ctemische Sammelliteratur Hr. P. Jacobson. 